\title{
Golgi Membrane Vesicles in HeLa Mitotic Cells are Identified with Monoclonal Antibody Made against Golgi Cisternal Membrane Protein p138
}

\author{
Shinichi Asada and Tatsuo Yagura* \\ Laboratory of Life Science, Department of Chemistry, Faculty of Science, Kwansei Gakuin University, \\ Nishinomiya, Hyogo 662, Japan
}

Key words: Golgi apparatus/mitosis/Golgi cluster/membrane vesicle/monoclonal antibody

\begin{abstract}
$A B S T R A C T$. A monoclonal antibody (mAbG3A5) recognizing p138 antigen was used to identify the Golgi cisternal membrane and determine behavior of Golgi fragments during mitosis in HeLa cells. At the start of mitosis, Golgi stacks identified with the mAbG3A5 antibody were fragmented into fine membrane vesicles which were distributed throughout the cytoplasm leaving only the region of the chromosome cluster unoccupied. On Western immunoblotting analysis, $\mathbf{p 1 3 8}$ was found associated with the membrane fraction prepared from mitotic HeLa cells having a buoyant density the same as that of interphase Golgi membranes. In addition to the fine membrane vesicles, clusters labeled with mAbG3A5 antibody were frequently observed in mitotic cells. They numbered 11 on average per mitotic cell and consisted of fine membrane vesicles of which membrane region was labeled with the mAbG3A5 antibody. This fact indicates that the membrane vesicles in mitotic Golgi clusters were also part of the fragments of Golgi cisternae. The number of mitotic Golgi clusters per mitotic cell was constant from prophase to anaphase, increasing twofold at telophase, although the average size of mitotic Golgi cluster remained unchanged throughout mitosis. The increase in number of mitotic Golgi clusters at telophase was accompanied by decrease in immunofluorescence of fine membrane vesicles. Treatment with nocodazole caused the disappearance of the mitotic Golgi clusters from prophase cells; however upon removal of it, they were reformed. These results suggest that during mitosis the Golgi apparatus were fragmented to fine membrane vesicles leaving only a part as mitotic Golgi clusters and were reassembled through tentative clustering of the fine membrane vesicles at the end of mitosis.
\end{abstract}

The Golgi apparatus of mammalian cells is comprised of several stacks arranged in a circumscribed juxtanuclear region to which the microtubule organizing center localizes (Thyberg and Moskalewski, 1985). Studies using electron microscopy and immunocytochemical procedures have shown that Golgi apparatus are fragmented and dispersed throughout the cytoplasm in mitotic cells (Zeligs and Wollman, 1979; Lucocq et al., 1987). The fragmentation of Golgi apparatus during mitosis would ensure equal partitioning of this organelle to the two daughter cells (Lucocq and Warren, 1987). In mitotic HeLa cells several clusters are shown comprised of a group of vesicles and tubules positive for the Golgi marker, galactosyltransferase (Lucocq et al., 1987 ) in addition to the dispersed free vesicles (Lucocq et al., 1989). These Golgi clusters are thought to serve as a template for rebuilding of the Golgi stack during telophase (Lucocq and Warren, 1987). However, a recent study by Tooze and Hollinshead (1992) suggests that most, if not all, of the Golgi clusters observed in mitotic HeLa cells are tubular early endosomes. Following this

\footnotetext{
* To whom correspondence should be addressed.
}

study, however, Pypaert et al. (1993) have again shown that their Golgi clusters were distinguished from the tubular endosomes using antibodies to two separate Golgi markers.

In this study we have readdressed the mitotic form of the Golgi apparatus using a novel monoclonal antibody against p138, a resident protein of Golgi cisternal membrane region. We have shown a large number of fine membrane vesicles as well as the existence of a small number of Golgi clusters in mitotic HeLa cells and suggest that an end form of Golgi cisternal membrane resulting from Golgi fragmentation is fine membrane vesicles, not mitotic Golgi clusters. However, during telophase of mitosis the membrane vesicles reassembled to form Golgi stacks via the clusters scattered throughout the cytoplasm.

\section{MATERIALS AND METHODS}

Antibodies. Hybridoma cell line (mAbG3A5) that secreted monoclonal antibody against p138 of human origin was prepared as described previously (Yamauchi et al., 1992). The monoclonal antibodies were obtained from ascitic fluids 
of mice inoculated with hybridoma cells and purified by chromatography on columns of affi-Gel protein A according to manufacturer's directions (Bio-Rad).

Immunoperoxidase electron microscopy. HeLa cells grown in minimal essential medium supplemented with $5 \%$ (vol/vol) calf serum on coverslips were fixed in situ with $2 \%$ paraformaldehyde in phosphate buffered saline (PBS) $(\mathrm{pH}$ 7.4) for $30 \mathrm{~min}$ at room temperature. After washing three times in PBS, the cells were permeabilized by treatment with $0.05 \%$ saponin in PBS containing $0.01 \%$ galatin for $10 \mathrm{~min}$. The permeabilized cells were incubated with the mAbG3A5 antibody $(20 \mu \mathrm{g} / \mathrm{ml})$ in PBS containing $0.05 \%$ saponin and $0.01 \%$ gelatin for $1 \mathrm{~h}$, washed three times in $0.05 \%$ saponinPBS over a 10 min period, then treated with horseradish peroxidase conjugated rabbit anti-mouse antibody $(26 \mu \mathrm{g} / \mathrm{ml})$ in PBS containing $0.05 \%$ saponin and $0.01 \%$ gelatin for $1 \mathrm{~h}$. The cells were washed three times in PBS, followed by staining in $0.1 \mathrm{M}$ Tris- $\mathrm{HCl}$, pH 7.6 containing $1 \mathrm{mg} / \mathrm{ml} \mathrm{DAB}$ and $0.01 \% \mathrm{H}_{2} \mathrm{O}_{2}$ for $30 \mathrm{~min}$ in the dark. The stained cells were infiltrated with epoxy resin as described previously (Takasuga and Yagura, 1993). Sections were examined in a JEOL JEM-100S electron microscope with no further heavy metal staining.

Western immunoblotting. Immunoblots were prepared by electrophoresing protein samples on minigels, transferring them to PVDF-membrane, and labeling them with the mAbG3A5 antibody and BCIP/NBT as described previously (Kozu et al., 1990).

Immunofluorescence microscopy and laser confocal microscopy. HeLa cells were grown on coverslips and fixed with 2\% paraformaldehyde in PBS for $30 \mathrm{~min}$ at room temperature. After three washings with PBS, cells were permeated with $0.5 \%$ Triton X-100 in PBS for $10 \mathrm{~min}$. The treated cells were next incubated with $20 \mu \mathrm{g} / \mathrm{ml}$ mAbG3A5 antibody for 1 $\mathrm{h}$ at room temperature. The cells were further treated for immunofluorescence microscopy as described earlier (Yamauch et al., 1992). Specimens were observed and photographed with an Olympus fluorescence microscope or an Olympus high-speed beam scanning laser confocal microscope.

Cell synchronization. HeLa cells were synchronized by the method of Zieve et al. (1980) using thymidine block and nocodazole. Mitotic cells were detached from culture dishes by pipetting. Greater than $96 \%$ of the cells were mitotic as determined by staining with Bis-Benzamide Hoechst 33258. In another experiment, mitotic cells were simply prepared from log phase HeLa cell cultures by pipetting procedure with no prior cell synchronization. More than $75 \%$ of the cells were mitotic. The cells remained on the petri dishes provided the interphase cells.

For release from the block at pro-metaphase, the cells were rinsed once with medium to remove nocodazole and then left to incubate in drug-free medium.

Assay of mannosidase II and $\alpha$-glucosidase activities. The activity of mannosidase II was assayed by measuring the hydrolysis of 4-metyl umberifferyl $\alpha$-D-mannopyranoside (Tulsiani et al., 1977). A $10 \mu$ l aliquot of each fraction was in- cubated with $2 \mathrm{mM}$ substrate in $100 \mu \mathrm{l}$ of $150 \mathrm{mM}$ sodium acetate (pH 5.7) for $30 \mathrm{~min}$ at $37^{\circ} \mathrm{C}$. After addition of $1.5 \mathrm{ml}$ stop solution $\left(0.133 \mathrm{M}\right.$ glycine/ $0.677 \mathrm{M} \mathrm{NaCl} / 0.083 \mathrm{M} \mathrm{Na}_{2} \mathrm{CO}_{3}$, $\mathrm{pH}$ 10.7), fluorescence of samples $(\mathrm{Ex}=360 \mathrm{~nm}, \mathrm{Em}=450$ $\mathrm{nm})$ was measured.

$\alpha$-Glucosidase activity was measured by the hydrolysis of $p$ nitrophenyl $\alpha$-D-glucopyranoside (Hino and Rothman, 1985). Aliquot of sample $(20 \mu \mathrm{l})$ was added to $500 \mu \mathrm{l}$ of $4 \mathrm{mM} p$-nitrophenyl $\alpha$-D-glucopyranoside in $50 \mathrm{mM}$ Hepes, $\mathrm{pH} 6.8 / 1 \%$ sodium cholate, and then incubated for $2 \mathrm{~h}$ at $30^{\circ} \mathrm{C}$. After addition of $1 \mathrm{ml}$ of $0.64 \%$ ethylen-diamine ( $\mathrm{pH} 10.7), \mathrm{OD}_{400}$ of the reaction product was measured.

Subcellular fractionations. Golgi membrane-rich fractions were prepared as described previously (Yamauch et al., 1992).

\section{RESULTS}

Localization of p138 to Golgi cisternal membrane in interphase HeLa cells. As reported earlier, p138 is localized to the Golgi membrane fraction, as previously revealed by subcellular fractionation procedure (Yamauchi et al., 1992). To identify the structure of the Golgi apparatus it is found on, p138 was visualized by immunoelectron microscopy using mAbG3A5 antibody. Fig. 1 shows that Golgi cisternae were labeled with antibody by the peroxidase reaction in interphase HeLa cell. The labels were localized to the membrane region of Golgi cisternae and no labeling was observed in the lumen of Golgi stacks (arrowheads in Fig. 1).

Next, we localized p138 in HeLa cell Golgi cisternae. As shown in Fig. 1, most of the recognizable Golgi cisternae were heavily labeled. However, in well-oriented sections, the most distal face of the Golgi stack was unlabeled (Fig. 2, arrows), while proximal and medial Golgi cisternae were intensely labeled. Associated vesicles adjacent to distal cisterna were negative for the labeling (Fig. 2A and B, arrowheads). Thus, p138 was shown to be a resident protein of cis and medial Golgi cisternal membrane and can be used to localize Golgi membranes in complicated cell systems such as the mitotic cell.

Fragmentation and reassembly of Golgi apparatus during mitosis. Analysis of the Golgi apparatus during mitosis was performed using mAbG3A5 antibody. Fig. 3A reveals an asymmetrical perinuclear localization of the Golgi apparatus in interphase HeLa cells labeled with the mAbG3A5 antibody. On observation of serial image sections made with confocal laser scanning microscopy, the p138-associated Golgi cisternae appeared to be an interconnected tubular network. Immediately after entering the mitotic phase, the Golgi apparatus was fragmented into fine structures that appeared as particulate matter distributed throughout the cytoplasm, leaving only the region of the chromosome 


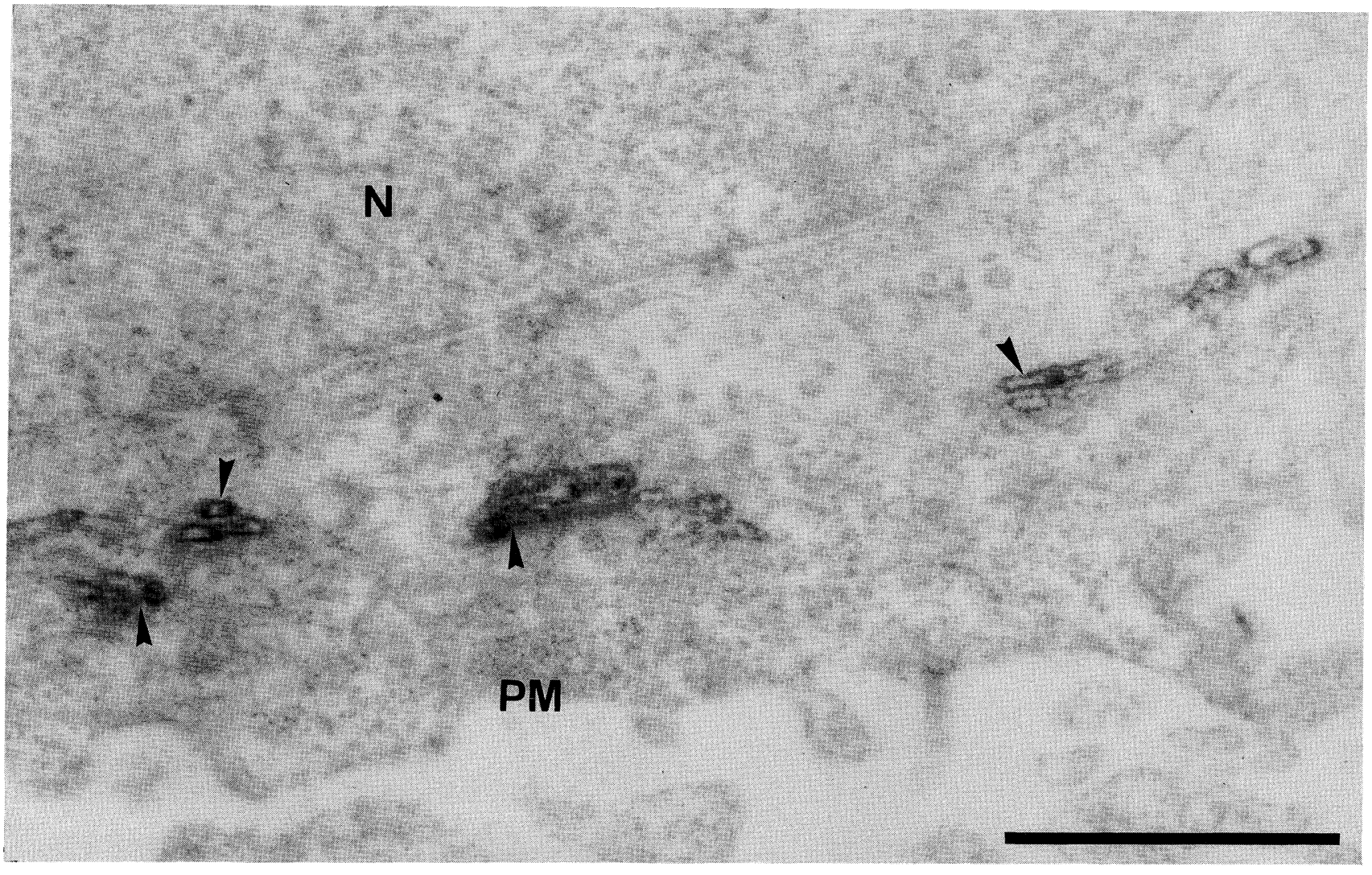

Fig. 1. p138 Labeled with mAbG3A5 antibody localized to Golgi apparatus membrane region in interphase HeLa cell. HeLa cells in random culture were fixed, permeabilized with saponin, and labeled with mAbG3A5 antibody followed by HRP-conjugated rabbit antimouse IgG. After reaction with diaminobenzidine hydrochloride (DAB) and $\mathrm{H}_{2} \mathrm{O}_{2}$, the cells were prepared for electron microscopy. Arrowheads indicate $\mathrm{HRP}$ labeled Golgi cisternal membranes. Bar, $1 \mu \mathrm{m}$. Abbreviations: N, nucleus; PM, plasma membrane.

cluster apparently unoccupied (Fig. 3B). This pattern of cytoplasmic distribution was not altered until the cells entered the telophase stage of mitosis.

In the mitotic cells a few distinct structures of larger size which were labeled by the antibody were also observed (arrows in Fig. 3C and D). Structures of the same size became more clearly visible and increased in number in anaphase to telophase (Fig. 3E and F), and then appeared to accumulate around the perinuclear region at cytokinesis (Fig. 3G). This was unquestionably observed in experiments using mAbG3A5 antibody purified using p138 immobilized on nitrocellulose membrane (data not shown). This structure resembled the Golgi cluster which was described by Lucocq et al. (1987). Thus we will refer to them as mitotic Golgi clusters in this paper.

Mitotic Golgi clusters. To further examine the profile of mitotic Golgi clusters, we took confocal images divided at $1.0 \mu \mathrm{m}$ intervals on the $z$-axis of anaphase cells (Fig. 4). The mitotic Golgi clusters apparently consisted of fine particulate structures (arrows in Fig. 4) closely resembling the fine particulate structures scat- tered throughout the cytoplasm. From successive images, it was clear that the mitotic Golgi clusters consisted of particulate structures, not tubular structures.

To study the fine particulate structures of the mitotic Golgi clusters and localization of p138, we performed immunoelectron microscopy experiments. As shown in Fig. 5A, p138 was found in the mitotic Golgi clusters. Each Golgi cluster was comprised of a group of membrane vesicles of $20 \mathrm{~nm}$ average in diameter (arrows in Fig. 5B). The membrane regions of vesicles, not lumenal regions, were labeled by mAbG3A5 antibody. A number of fine membrane vesicles were occasionally observed with labeled membrane. These fine membrane vesicles appeared clustered in small regions, not evenly distributed in cytoplasm. However, DAB signals were quite weak on these structures, thus at present we could not identify them as the fine particulate structure observed in Figs. 3 and 4. The membrane vesicles constituting the mitotic Golgi clusters were mainly round in shape, and tubular vesicles were scarcely observed. In this experiment, we did not observe any organelle structures, such as endoplasmic reticulum, surrounding the 
mitotic Golgi cluster.

On detailed observation of mitotic cells immunostained by the mAbG3A5 antibody with confocal laser microscope, each stage of mitotic cells had clusters of average $700 \mathrm{~nm}$ in diameter, though this size is probably

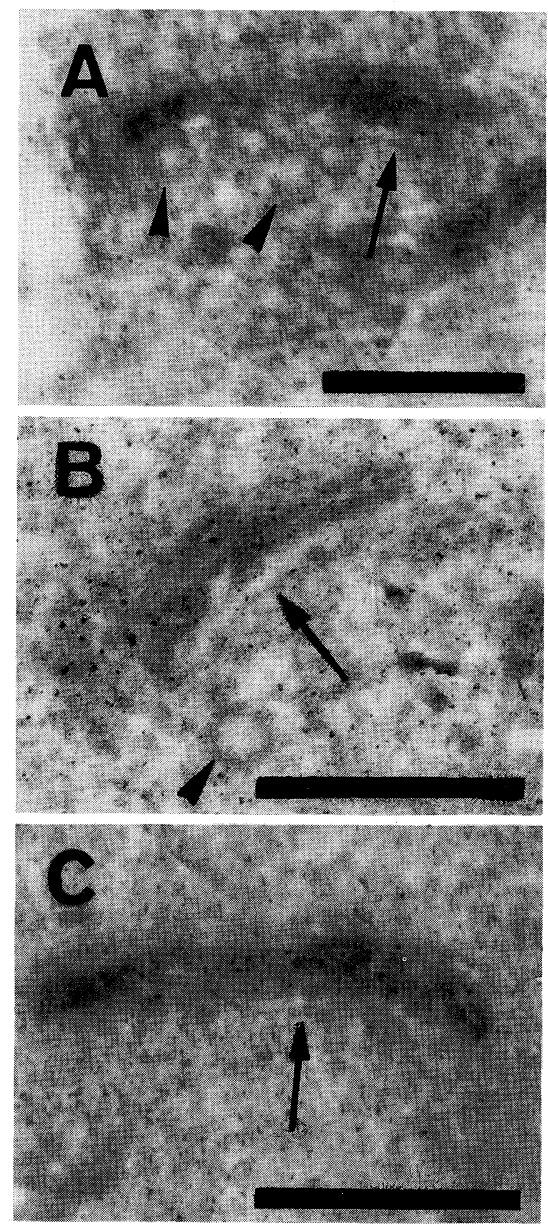

Fig. 2. Immunoperoxidase labeling of Golgi stacks with mAbG3A5 antibody. HeLa cells were processed for immunoelectron microscopy as described under Materials and Methods. Thin sections were viewed after staining with lead citrate. Unlabeled single cisternae with immunoperoxidase reaction were indicated by arrows. Arrowheads denote a part of unlabeled small vesicles. Bar, $0.5 \mu \mathrm{m}$.

Fig. 3. Fragmentation and reconstruction of the Golgi complex during mitotic phase of HeLa cells detected with the mAbG3A5 antibody. HeLa cells in log phase culture were fixed, permeabilized and stained with mAbG3A5 antibody. Bound antibody was visualized by secondary antibody coupled to FITC viewed under a laser scanning confocal microscope (A-G). H-N show DNA stained with Hoechst 33258 in corresponding cells, photographed with a fluorescence microscope. (A, H), interphase; (B, I), prophase; (C, J), metaphase; (D, $\mathrm{K})$, anaphase; (E, L), telophase; (F, M, G, N), cytokinesis. Note the reconstruction of the Golgi complexes at opposite regions of the face of cell fission in G. Arrows in C and D indicate the Golgi clusters. Bar, $10 \mu \mathrm{m}$. overestimated because of fluorescence halo. The number and size distribution were obtained for mitotic Golgi clusters from serial section analyses using confo-
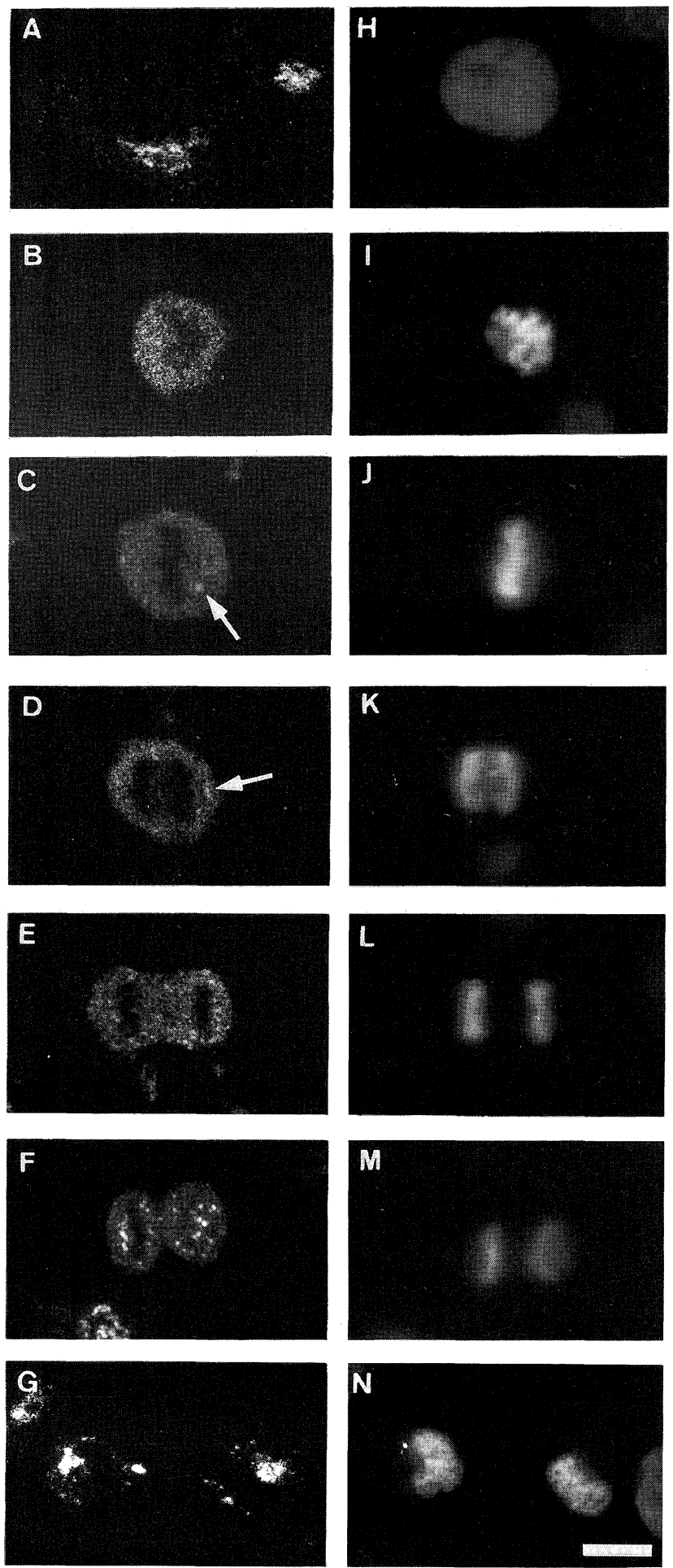

Fig. 3. 

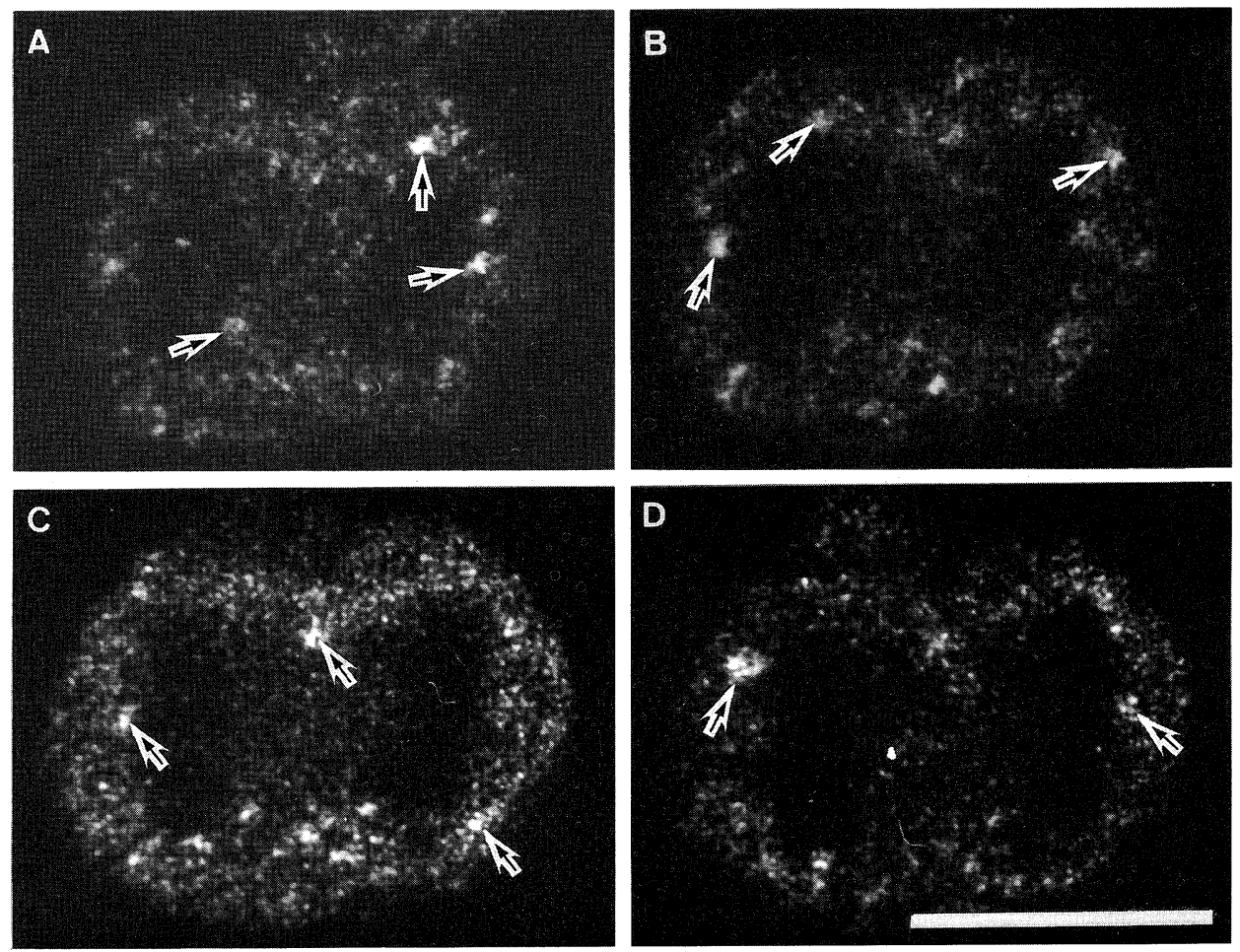

Fig. 4. Image analysis of mitotic cell labeled by mAbG3A5 antibody with confocal laser scanning microscope. Mitotic HeLa cell was stained with mAbG3A5 antibody as described in Materials and Methods. The cell on the coverslip was analyzed with a confocal laser scanning microscope at $1.0 \mu \mathrm{m}$ sectioning on the $z$-axis from the top of cell to the adherent side of cell (A-D). The sectioning was performed at midregion of the cell and other portions were not shown. Arrows indicate a part of typical Golgi clusters. Bar, $10 \mu \mathrm{m}$.
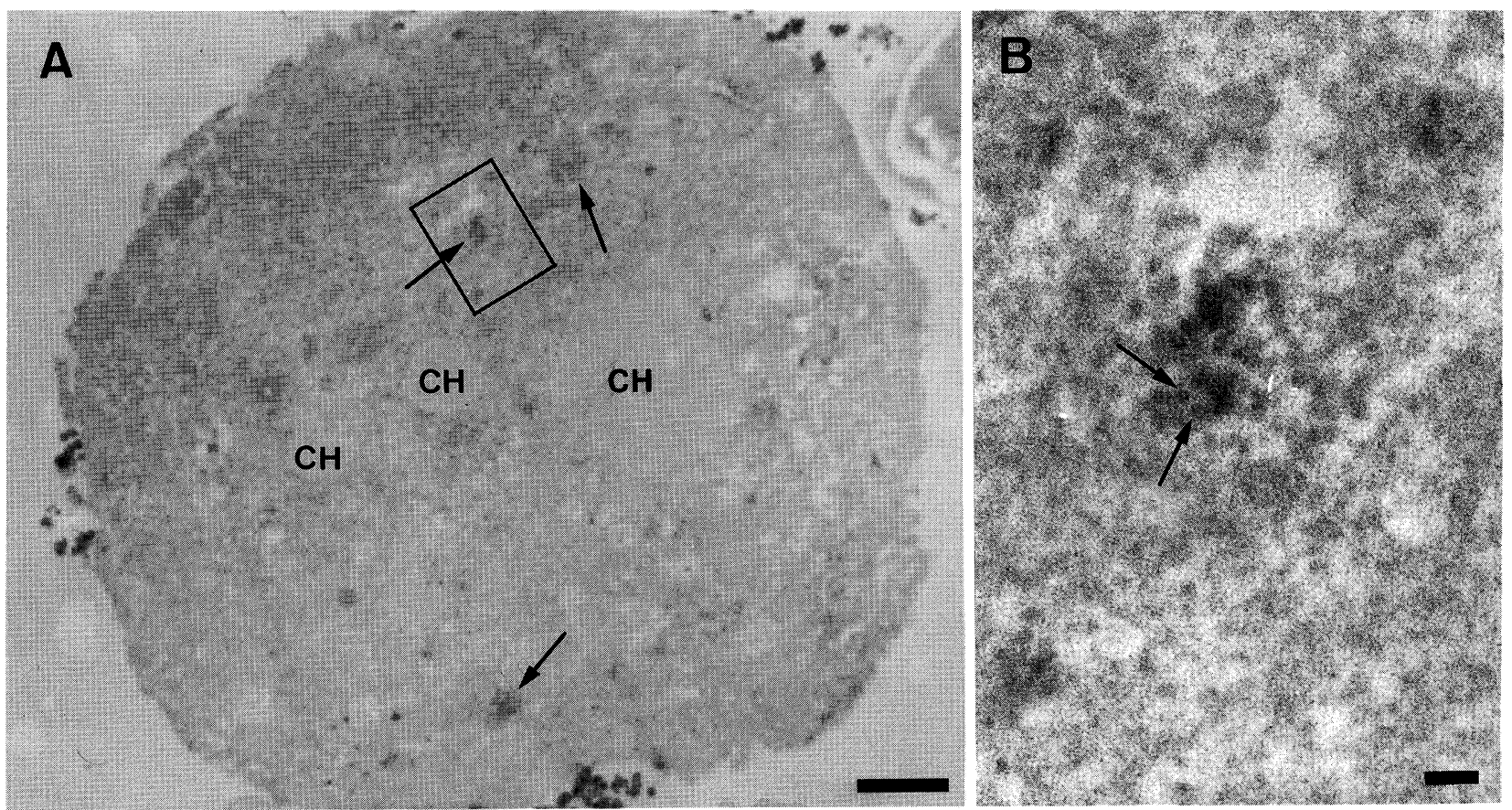

Fig. 5. Electron microscopy of Golgi clusters labeled with mAbG3A5 antibody of mitotic HeLa cell. Mitotic HeLa cells were treated as described in Fig. 1 legend. In A, metaphase cell stained with mAbG3A5 antibody is shown containing several Golgi clusters (arrows). A higher magnification micrograph of the area within box in A is shown in B. This region contains a Golgi cluster which comprises several vesicular structures stained with mAbG3A5 antibody (arrows in B). $\mathrm{CH}$, mitotic chromosome. Bar, $1 \mu \mathrm{m}$ in $\mathrm{A}$ and $0.1 \mu \mathrm{m}$ in $\mathrm{B}$. 


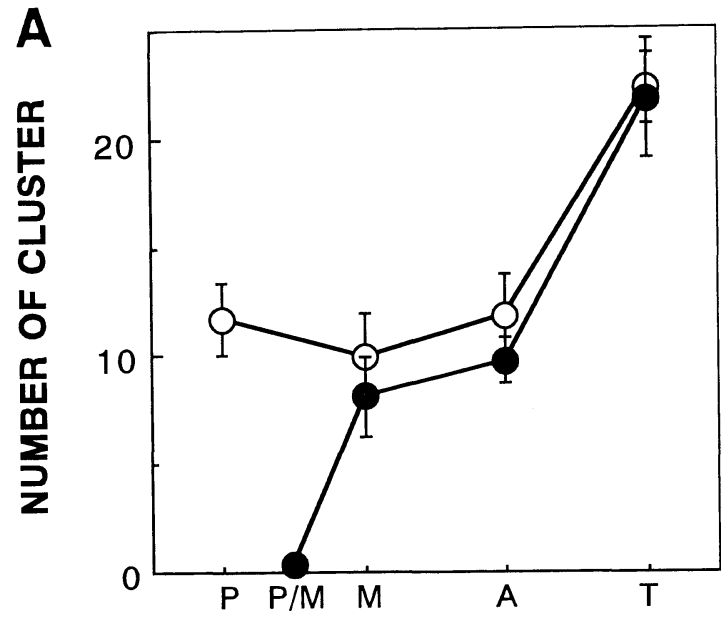

PHASE

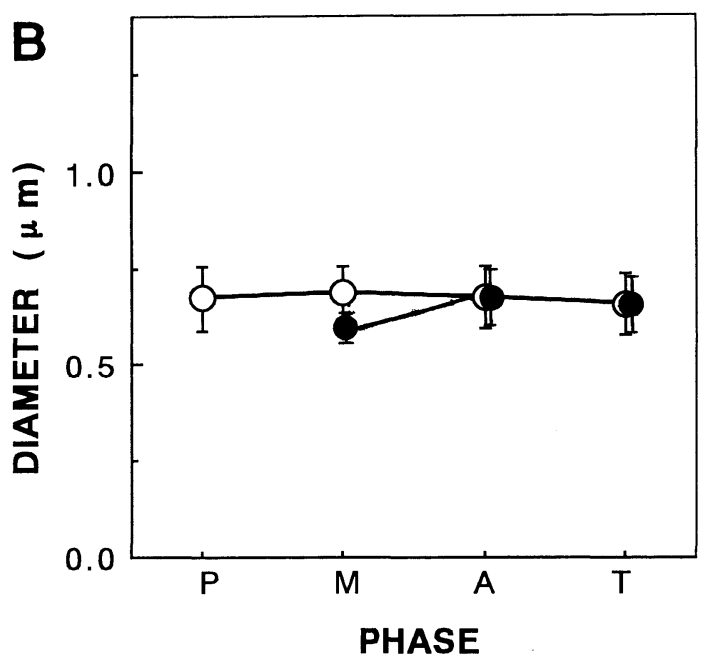

Fig. 6. Changes of Golgi cluster in number and size during progression of mitosis and the effect of nocodazole-pretreatment. HeLa cells at $\log$ phase were labeled with the mAbG3A5 antibody followed by FITC conjugated sheep antimouse second antibody. Using image analysis by laser scanning confocal microscope, the number (A) and size (B) of Golgi clusters were determined in mitotic cells. In nocodazole experiment, HeLa cells in log phase were synchronized to pro-metaphase as described in Materials and Methods. After release from nocodazole blocking by washing with fresh MEM supplemented with $10 \%$ fetal bovine serum, the mitotic cells were left to incubate in drug-free medium, and were then labeled as above and examined with the laser scanning confocal microscope. $(\bigcirc)$, mitotic cells; $(\bullet)$, mitotic cells obtained after synchronization with nocodazole treatment. Abbreviations: P, prophase; P/M, pro-metaphase; M, metaphase; A, anaphase; $\mathrm{T}$, telophase.

cal laser scanning microscope. As shown in Fig. 6A, the number of mitotic Golgi clusters per mitotic cell was steady at an average of 10 to 11 during pro-metaphase to anaphase and increased to an average of 22 at telophase. On the other hand, the average diameter of

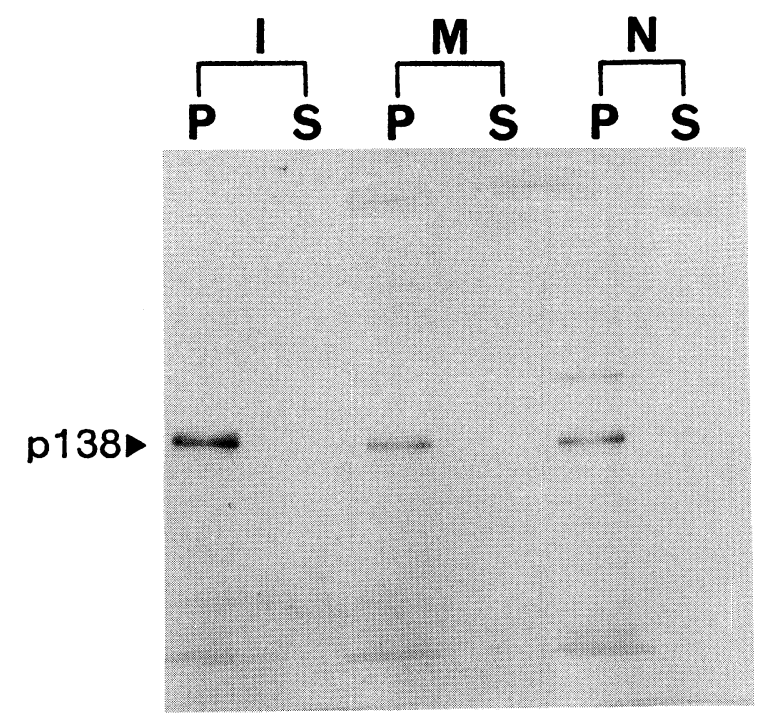

Fig. 7. Association of the p138 antigen with microsomal fraction containing Golgi membrane prepared from the pro-metaphase of nocodazole treated culture. HeLa cells in interphase and mitotic phase were disrupted and fractionated as described in Materials and Methods. The microsomal pellet $(\mathrm{P})$ and its supernatant fraction $(\mathrm{S})$ were analyzed by Western immunoblotting with mAbG3A5 antibody. (I), interphase cells; $(\mathrm{M})$ and $(\mathrm{N})$, mitotic cells obtained by use of mitotic pipetting procedure from random culture and nocodazole-synchronized culture, respectively.

clusters remained constant throughout metaphase (Fig. 6B).

Mitotic Golgi clusters are maintained with microtubules. The Golgi apparatus in interphase cells maintains its structure by microtubules constituting the cytoskeleton (Thyberg and Moskalewski, 1985). In order to examine participation of microtubules in the formation of mitotic Golgi clusters in mitotic cells, HeLa cells were incubated in the presence of nocodazole after presynchronization treatment with excess thymidine, then pro-metaphase cells were stained with the mAbG3A5 antibody. As shown in Fig. 6A (P/M), no mitotic Golgi clusters were observed. However, upon release from the nocodazole block, the Golgi clusters reappeared. The number of Golgi clusters in recovered mitotic cells were rapidly restored to the level of non-treated mitotic cells (Fig. 6A). The size distribution of Golgi clusters in recovered cells was almost the same as that in non-treated mitotic cells (Fig. 6B). Thus, microtubules, which were destabilized by nocodazole, were shown to have an effect on the clustering of Golgi membrane vesicles.

Fine particulate structures in mitotic cells are membrane vesicles. The major portion of fluorescence labeling by the mAbG3A5 antibody in mitotic cells, except for telophase cells, was localized to the fine particulate structures. On immunochemical microscopy (Fig. 4), a number of antibody-labeled fine particulate struc- 


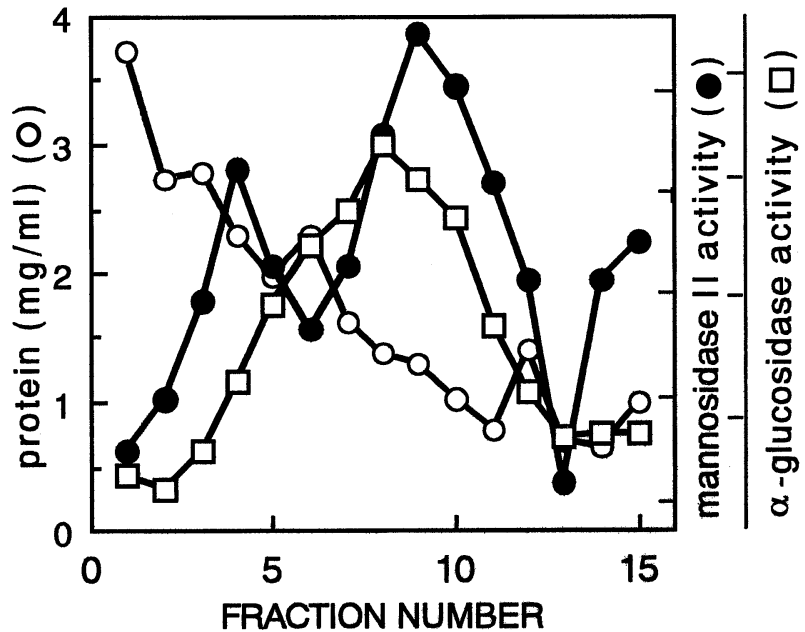

$\begin{array}{lllllllllllllll}M & 3 & 4 & 5 & 6 & 7 & 8 & 9 & 10 & 11 & 12 & 13 & 14 & 15\end{array}$

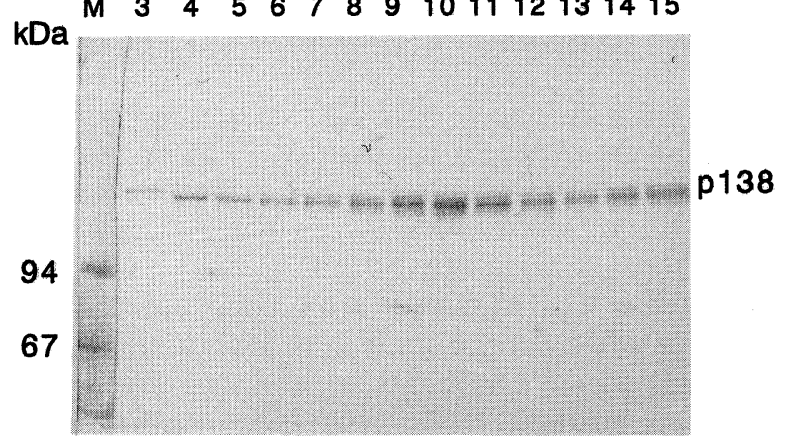

$\mathbf{A}$
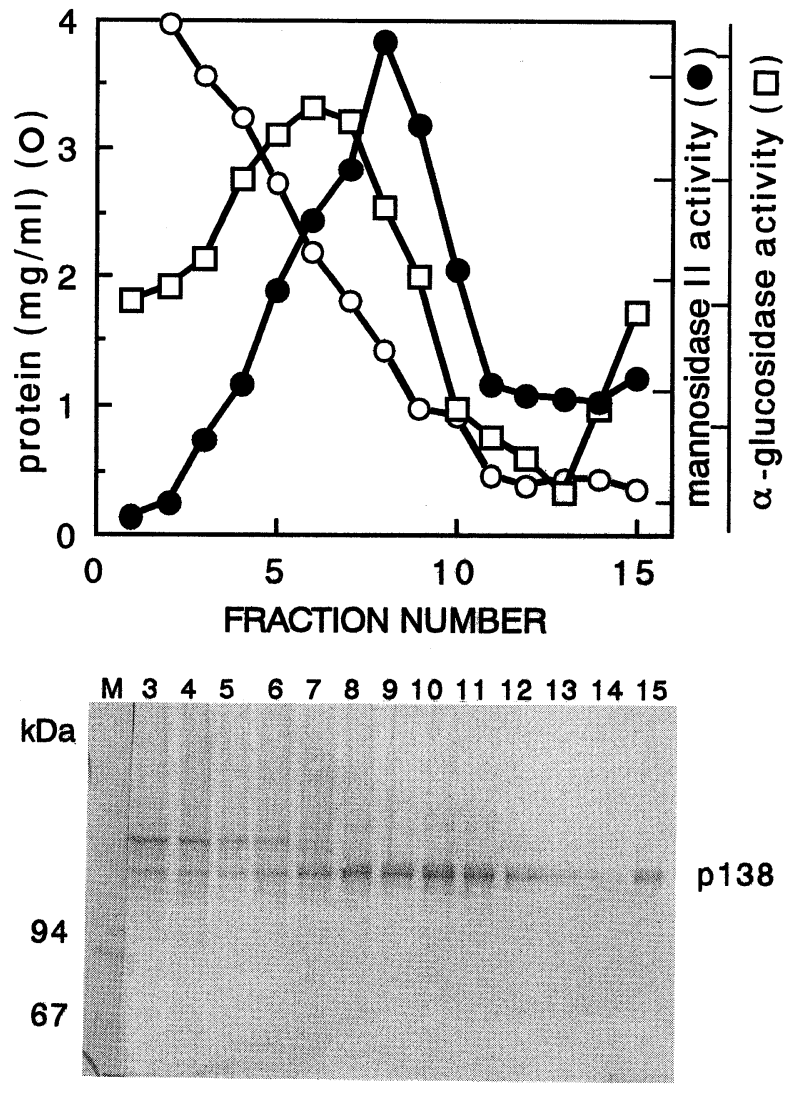

B

Fig. 8. The membrane fraction associated with p138 prepared from pro-metaphase cells of nocodazole-synchronized culture has the same density as that of the Golgi cisternal membrane. Microsomal membranes in $100,000 \times \mathrm{g}$ pellet fraction were separated by flotation in $20-45 \%$ sucrose gradient (Yamauchi et al., 1992). After centrifugation at $150,000 \times \mathrm{g}$ for $8 \mathrm{~h}$ in a Hitachi RP-55S rotor, $140 \mu \mathrm{l}$ portions for each tube were collected from the bottom. (A), microsomal membranes prepared from interphase HeLa cells; (B), microsomal membranes from pro-metaphase cells of nocodazole synchronized culture. The lower panels of (A) and (B) represent Western immunoblotting with the mAbG3A5 antibody of each fraction of upper graphs. The protein band of higher molecular weight was seen on Western immunoblotting shown in B, in addition to the p138 band. At present we do not know whether this indicates the presence of another protein weakly reactive with mAbG3A5 antibody because the occurrence and amount of this protein band were variable from experiment to experiment. As seen in B, this protein sedimented at the bottom. It suggests that this protein is not associated with membrane and thus is not Golgi resident membrane protein.

tures were observed scattered throughout the cytoplasm of mitotic cells. As pointed out, the fine particulate structure was shown to decrease in number from anaphase to telophase parallel with the increase in number of the mitotic Golgi clusters (see Fig. 3). Thus it is possible that the fine particulate structures are an end form of fragmented Folgi cisternal membranes.

To test that possibility, we examined whether the fine structures was membranous structures. Mitotic cells were obtained by mitotic pipetting method after synchronization of the culture of HeLa cells using nocodazole to completely destabilize the mitotic Golgi cluster. Then, fractionation was performed for the preparation of Golgi membrane-rich fraction. As shown in Fig. 7, p138 in mitotic cells synchronized by nocodazole was recovered on the Golgi membrane-rich fraction $(\mathrm{N})$. We also obtained mitotic cells by the mitotic pipetting method of an exponentially growing culture with no prior cell synchronization, though a decreased yield. The p138 thus obtained was also localized to the Golgi membrane-rich fraction (Fig. 7M). These results suggest that most of p138 in mitotic cells treated with nocodazole localizes to membrane structures, and is not present as free protein in the cytoplasm. To further test that hypothesis, we studied the density of the structures containing p138 by use of sucrose density gradient centrifugation. Fig. 8B shows that p138 equilibrated at the density of $=1.388$, which closely coincided with that of Golgi membrane fraction of interphase cells (see Fig. 8A) and mannosidase II, a Golgi resident enzyme (Goldberg and Kornfeld, 1983). Thus, p138 is suggested to be associated with membranous structures with a den- 

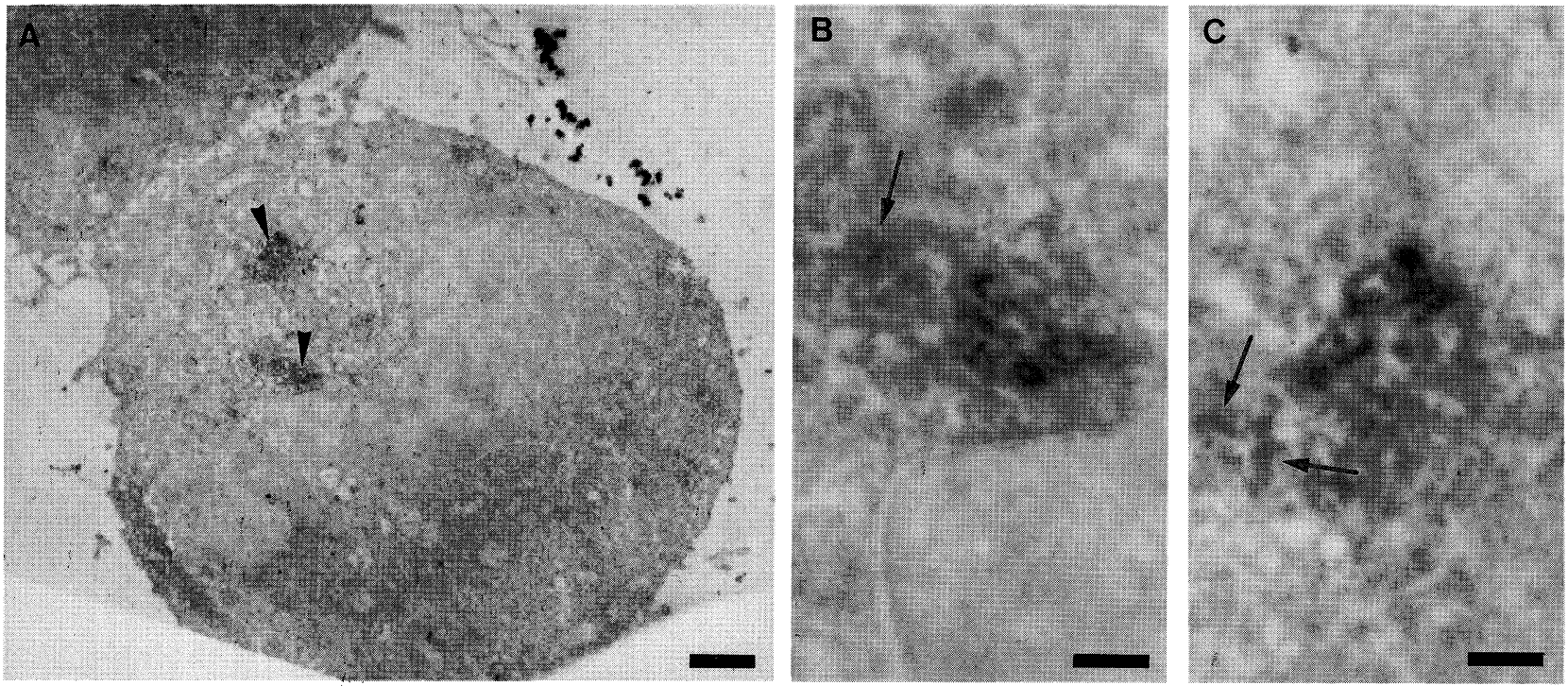

Fig. 9. Morphology of reassembling Golgi stacks in late anaphase cell labeled with the mAbG3A5 antibody. The mitotic cells obtained by mitotic pipetting procedure from the $\log$ phase culture of HeLa cells were labeled with the mAbG3A5 antibody as described in Fig. 1 legend. (A), cell at cytokinesis; (B) and (C), higher magnification micrographs of reassembling Golgi stack area in (A) (arrowheads). Arrows in B and C show tubular vesicles. Bars, $1 \mu \mathrm{m}$ in (A) and $0.2 \mu \mathrm{m}$ in (B) and (C).

sity identical to that of Golgi membrane of interphase cells.

Golgi stacks reassemble during telophase via fusion of round vesicles associated with p138. At telophase, the number of Golgi clusters increased and they appeared to assemble to the juxtanuclear region upon cytokinesis (see Fig. 3). Thus we next studied whether the membrane vesicles were fused to each other in the clusters at cytokinesis.

Fig. 9 shows the immunoelectron microscopy of cells from telophase to cytokinesis labeled by the mAbG3A5 antibody. Two pre-Golgi apparatus were seen near the nuclear envelope (arrowheads). In magnified pictures of pre-Golgi region (Fig. 9B and C), it was evident that the pre-Golgi region consisted of clusters of membrane vesicles of various sizes from $20 \mathrm{~nm}$ to $90 \mathrm{~nm}$. The size distribution of membrane vesicles was apparently larger than that of membrane vesicles of the fine particulate structures and the mitotic Golgi clusters. Most of the vesicles were round in shape, although some tubular vesicles were also present in the pre-Golgi region (Fig. $9 \mathrm{~B}$ and $\mathrm{C}$, arrows). The round shape of membrane vesicles was not ascribed to artificial effect of the treatment with saponin because all the Golgi apparatus in interphase cells contaminated in the same sample presented the typical tubular structure as shown in Fig. 1.

\section{DISCUSSION}

In this work, we have used the monoclonal antibody against p138 and described the mitotic membrane vesi- cles associated with p138 derived from Golgi apparatus. Partial nucleotide sequence analysis of cDNA clones of p138 have shown no homology with mouse mannosidase II (Moremen and Robbins, 1991) or any other proteins reported up to now. We have shown in this study that the cisternal membranes associated with p138 retain their identity during fragmentation and reassembly of Golgi apparatus. Thus we can use this Golgi membrane resident protein as a new marker of the Golgi apparatus.

Several characteristics of our mitotic Golgi clusters resembled those reported by Lucocq et al. (1987). Their study showed that their mitotic Golgi cluster was comprised of a group of large electron-lucent vesicles up to $250 \mathrm{~nm}$ in diameter, and a group of smaller, vesiculotubular structures with a cross sectional diameter of 50 $\mathrm{nm}$. Our mitotic Golgi clusters however averaged 260 $\mathrm{nm}$ in diameter and were comprised of a group of homogeneous round vesicles of $20 \mathrm{~nm}$ in diameter on observation by immunoelectron microscopy (see Fig. 5). Furthermore, the number of our mitotic Golgi clusters per early mitotic cell was 10-11 and was not restricted to the peripheral region of the cells, whereas Lucocq et al. presented a larger number (10 to 300 clusters) with the occurrence of Golgi clusters mainly at the periphery of mitotic cells (Lucocq and Warren, 1987; Lucocq et al., 1987). These inconsistencies from the results of Warren's group may be attributed to the difference in the localization of markers used. To label the vesicular and tubular structures in the Golgi clusters, Pypaert et al. (1993) used antibodies against two resident Golgi 
markers, one restricted to the trans cisternae and the trans-Golgi network and the other marking the medial cisternae, whereas we used antibody against p138 which was restricted to the cis and medial cisternae. In addition, the attempt to stain Golgi membranes in mitotic cells is very difficult because of traffic of these organelle membranes among other organelles, and as pointed out by Tooze and Hollinshead (1992), mitotic HeLa cells contain tubular early endosomes morphologically indistinguishable from Golgi clusters reported by Lucocq et al. (1987). Thus the comparison of the results obtained by experiments using different markers might be of value for the better understanding of mechanism controlling fragmentation and reassembly of Golgi complex during mitotic cycle.

In addition to the fact of destabilization of Golgi clusters by nocodazole treatment, a large number of fine membrane vesicles at early to mid-mitotic stage make us favor the idea that these vesicles are an endproduct of fragmentation of Golgi cisternal membranes. The mitotic Golgi clusters appearing at the same mitotic stage may remnants of fragmentation of Golgi cisternae. The number of mitotic Golgi clusters increased and was dispersed throughout the cytoplasm at telophase, followed by their return to the region around the microtubule-organizing center and reassembling of Golgi stacks. These facts and the morphologic change of membrane vesicles in clusters during telophase suggest that the mitotic Golgi clusters may serve as a template for the assembly of Golgi stack, as suggested by Lucocq and Warren (1987).

Acknowledgments. Part of this work was performed under Frontier Research Project in Telecommunications supported by Ministry of Posts and Telecommunications and Support Center for Advanced Telecommunications Technology Research.

\section{REFERENCES}

1. Goldberg, D.E. and Kornfeld, S. 1983. Evidence for extensive subcellular organization of asparagine-linked oligosaccharide processing and lysosomal enzyme phosphorylation. J. Biol. Chem., 258: 3159-3165.

2. Hino, Y. and Rothman, J.E. 1985. Glucosidase II, a glycoprotein of the endoplasmic reticulum membrane. Proteolytic cleavage into enzymatically active fragments. Biochemistry, 24: 800805 .

3. KozU, T., Nikaido, K., and Yagura, T. 1990. Structure of DNA polymerase-primase complexes from mammalian cells analyzed by using monoclonal antibodies. J. Biochem., 107: 536538.

4. Lucoce, J.M., Pryde, J., Berger, E., and Warren, G. 1987. A mitotic form of the Golgi apparatus in HeLa cells. $J$. Cell Biol., 104: 865-874.

5. LuCocQ, J.M. and WARren, G. 1987. Fragmentation and partitioning of the Golgi apparatus during mitosis in HeLa cells. EMBO J., 6: 3239-3246.

6. LucocQ, J.M., Berger, E.G., and Warren, G. 1989. Mitotic Golgi fragments in HeLa cells and their role in the reassembly pathway. J. Cell Biol., 109: 63-474.

7. Moremen, K.W. and Robiins, P.W. 1991. Isolation, characterization, and expression of cDNA encoding murin alpha mannosidase II, a Golgi enzyme that controls conversion of high mannose to complex N-glycans. J. Cell Biol., 115: 1521-1534.

8. Pypaert, M., Nilsson, T., Berger, E.G., and Warren, G. 1993. Mitotic Golgi clusters are not tubular endosomes. $J$. Cell Sci., 104: 811-818.

9. Takasuga, Y. and Yagura, T. 1993. Assembly of envelope structure with vesicles associated with $\mathrm{Ku}$-homologous protein in Xenopus egg extract in the absence of chromatin. Cell Struct. Func., 18: 205-210.

10. Thyberg, J. and Moskalewski, S. 1985. Microtubules and the organization of the Golgi complex. Exp. Cell Res., 159: 116.

11. Tooze, J. and Hollinshead, M. 1992. Evidence that globular Golgi clusters in mitotic HeLa cells are clustered tubular endosomes. Euro. J. Cell Biol., 58: 228-242.

12. Tulsiani, D.R.P., Opheim, D.J., and Touster, O. 1977. Purification and characterization of a $\alpha$-D-mannosidase from rat liver Golgi membranes. J. Biol. Chem., 252: 3227-3233.

13. Yamauchi, T., Higashiura, M., and Yagura, T. 1992. Novel monoclonal antibody mAbG3A5 recognizes $138-\mathrm{kDa}$ glycoprotein localized on the Golgi membrane. Cell Struct. Func., 17: 213-222.

14. Zeligs, J. and Wollman, S.H. 1979. Mitosis in rat thyroid epithelial cells in vivo. I. Ultrastructural changes in cytoplasmic organelles during the mitotic cycle. J. Ultrastruct. Res., 66: 3-77.

15. Zieve, G.W., Turnbull, D., Mullins, J.M., and McIntosh, J.R. 1980. Production of large numbers of mitotic mammalian cells by use of the reversible microtubule inhibitor nocodazole. Exp. Cell Res., 126: 397-405.

(Received for publication, July 28, 1995

and revised form, September 28, 1995) 